\title{
BMJ Open Acute interventions for aggression and agitation in psychosis: study protocol for a systematic review and network meta- analysis
}

\author{
Edoardo G Ostinelli (D) , ${ }^{1}$ Armando D'Agostino (D) , ${ }^{1}$ Farhad Shokraneh (D) ,2 \\ Georgia Salanti, ${ }^{3}$ Toshi A Furukawa ${ }^{4}$
}

To cite: Ostinelli EG, D'Agostino A, Shokraneh F, et al. Acute interventions for aggression and agitation in psychosis: study protocol for a systematic review and network meta-analysis. BMJ Open 2019;9:e032726. doi:10.1136/ bmjopen-2019-032726

- Prepublication history and additional material for this paper are available online. To view these files, please visit the journal online (http://dx.doi. org/10.1136/bmjopen-2019032726).

Received 02 July 2019 Revised 28 August 2019 Accepted 16 September 2019

Check for updates

(C) Author(s) (or their employer(s)) 2019. Re-use permitted under CC BY-NC. No commercial re-use. See rights and permissions. Published by BMJ.

For numbered affiliations see end of article.

Correspondence to Dr Edoardo G Ostinelli; eg.ostinelli@gmail.com

\section{ABSTRACT}

Introduction Individuals with psychosis may access emergency services due to aggression and agitation. When the de-escalation technique fails to achieve tranquillisation, several pharmacological options are available. However, evidence on which intervention to prefer in terms of efficacy and tolerability to achieve resolution of the acute episode (ie, rapid tranquillisation) of aggression and agitation is currently fragmentary. Methods and analysis We will include all randomised controlled trials comparing drugs or drug combinations or placebo for aggression or agitation episodes in adult individuals with psychosis. We will include individuals with psychosis (eg, schizophrenia and related disorders, bipolar disorder with psychotic symptoms, psychotic depression) but not substance or medication-induced psychosis or psychosis due to another medical condition. Our primary outcomes are the change in aggression or agitation scores within few hours since the administration of the intervention (efficacy outcome) and the proportion of participants who dropped out due to adverse effects (tolerability outcome). We will retrieve relevant studies from the register of studies of the Cochrane Schizophrenia Group. Also, we will run additional searches on CENTRAL, Embase and PubMed to retrieve potentially eligible studies focusing on other psychiatric diagnoses than those in the schizophrenia spectrum. We will conduct a random-effects network meta-analysis (NMA) for primary and secondary outcomes. In case of rare events of dichotomous outcomes, a common-effect Mantel-Haenszel NMA will be used instead. We will use the surface under the cumulative ranking curve and the mean ranks to rank all available treatments. Local and global methods of evaluation of inconsistency will be employed. Quality of evidence contributing to network estimates of the main outcomes will also be assessed with Confidence in Network MetaAnalysis.

Ethics and dissemination This study does not require ethical approval. We will disseminate our findings by publishing results in a peer-reviewed journal. PROSPERO registration number CRD42019137945.

\section{BACKGROUND}

Mental health-related behavioural emergencies are reported to account for around

\author{
Strengths and limitations of this study
}

- Our systematic review and random-effects network meta-analysis (NMA) will provide pragmatic answers to a critical question in the field of psychiatry: which drug or drugs combination, if any, is currently the best evidence-based choice in terms of efficacy and tolerability for rapid tranquillisation of aggression or agitation episodes in individuals with psychosis?

- The analysis will benefit from maximum statistical power by combining direct and indirect comparisons in an NMA, measuring the relative efficacy of these treatments.

- We will evaluate the contribution of drugs to their combination intervention by performing a component NMA as a sensitivity analysis.

- Limitations of individual studies will be judged with the revised Cochrane risk of bias tool $(\mathrm{RoB} 2)$ and the credibility of the results for the main outcomes will be assessed using the Confidencein Network MetaAnalysis web application.

- Some studies might have evaluated more pragmatic outcomes without a continuous measurement of the level of aggression or agitation, thus not being able to contribute our primary efficacy outcome.

$6 \%$ of the visits to the emergency department in USA. ${ }^{1}$ In this context, aggression and agitation are clinically relevant issues in both psychiatric and emergency department settings. ${ }^{2}$ Verbal and physical aggression may result from psychomotor agitation, of which restlessness, excitability and dysphoria are prominent symptoms. ${ }^{34}$ Violent behaviour has been reported to occur in $18.5 \%$ of participants with psychosis included in a recently published systematic review. ${ }^{5}$ Consequences of this phenomenon may affect patients, their caregivers, healthcare personnel and community members, both in terms of harmful events towards self or others and stigma-driven victimisation of patients. ${ }^{6-9}$ 
When facing acutely aggressive and agitated individuals, guidelines recommends clinicians to assess the feasibility of management with de-escalation techniques. Should this be deemed unpractical or proven ineffective, a pharmacological approach is the mainstay for rapid tranquillisation. ${ }^{10}{ }^{11}$ Several systematic reviews have evaluated the comparative efficacy and safety of pharmacological interventions from randomised controlled trials (RCTs) in schizophrenia spectrum illness. ${ }^{12-24}$ These reviews contributed currently available policy-making policies: for instance, NICE guidelines recommend the use of lorazepam or the combination of haloperidol and promethazine as pharmacological interventions for aggression and agitation in the context of schizophrenia spectrum illnesses. ${ }^{11}$

However, evidence supporting these recommendations is still fragmentary and a comprehensive ranking of all treatments evaluated in RCTs is still lacking. Also, several interventions lack of head-to-head comparisons with recommended and available treatment options. For example, none of the identified RCTs compared the intramuscular administration of the second-generation antipsychotic aripiprazole with either lorazepam or the haloperidol and promethazine combination. ${ }^{1120}$

Network meta-analysis (NMA) is a statistical technique that provides estimates through direct and indirect comparisons and allows to assess the relative ranking of different treatments, ultimately resulting in increased precision. ${ }^{25}$ Objective of this systematic review and NMA is to compare available pharmacological interventions in terms of efficacy and tolerability in the acute treatment of aggression and agitation episode (ie, rapid tranquillisation) in adults with psychosis presenting to an acute care setting. In order to ensure our findings to be pragmatic and to provide clinically meaningful recommendations, we will focus on the transdiagnostic concept of psychosis when not induced by substance or medication use or other medical conditions.

\section{METHODS}

We submitted this study protocol to PROSPERO (CRD42019137945). Methods for this systematic review and NMA have been developed following the Preferred Reporting Items for Systematic Review and Meta-Analysis (PRISMA) guideline for systematic review and meta-analysis protocols (PRISMA-P, online supplementary file 1), the extension statement for NMA (PRISMA-NMA, online supplementary file 2) and proposed additional considerations for protocols of systematic reviews including NMA. ${ }^{26-29}$

\section{Criteria for considering studies for this review \\ Types of studies}

RCTs reported as double-blind will be included. Singleblind and open studies will be included but the effect of their inclusion will be evaluated in a sensitivity analysis. Quasi-randomised studies (eg, allocating by using alternate days of the week) will be excluded. Cross-over and cluster randomised trials will be included. Studies where sequence generation was at high risk of bias or where allocation was clearly not concealed will not be included.

\section{Types of participants}

Individuals aged 18 years or older, of both sexes, showing psychotic symptoms and an aggression or agitation episode deemed to be in need of pharmacological tranquillisation. We will also include studies in which baseline mean scores of an aggression or agitation rating scale suggest the need of pharmacological tranquillisation (eg, Positive and Negative Syndrome Scale Excited Component (PANSS-EC) $\geq 14$, Modified Overt Aggression Scale (MOAS) $\geq 4)$. The impact of including these studies will be analysed (refer to the Exploring heterogeneity, inconsistency and sensitivity analyses section). Studies will be considered eligible only if psychotic symptoms are thought to be secondary to an established psychiatric diagnosis (eg, schizophrenia, schizophreniform disorder, schizoaffective disorder, delusional disorder, brief psychotic disorder, bipolar mania or depression with psychotic features, psychotic depression) in at least $50 \%$ of the randomised population. Psychotic disorders induced by substances or medications or due to another medical condition (eg, dementia, Parkinson's disease, autoimmune disorders, neurodevelopmental disorders) will not be considered eligible. Peri-partum psychosis will not be considered eligible. A concurrent secondary diagnosis of another psychiatric disorder will not be considered as exclusion criteria. Treatment settings may be the outpatient emergency departments or the hospital wards.

\section{Types of interventions}

We aim to synthesise available data for first-generation antipsychotics, second-generation antipsychotics, benzodiazepines, antihistaminic drugs and valproic acid or their combinations. We will obtain information about the interventions of interest from head-to-head and placebo-controlled trials (ie, pill or pharmacological placebo). Inclusion of placebo-controlled trials may contribute via indirect evidence to the treatment comparisons of interest by increasing the precision and allowing additional connections in the network. Pharmacological interventions not described above will be considered for post-hoc inclusion in the network within the context of the joint randomisability assumption by evaluation of their effect as rapid tranquilliser. Interventions (drugs or drug combinations) may be administered via any route of administration consistent with the rapid tranquillisation aim (ie, long-acting injectable antipsychotics are not eligible since they aim at maintenance treatment). Any dosing strategy (ie, flexible or fixed) will be allowed. We will allow multiple administrations and we will consider them when evaluating the eligibility of dosages and fairness of comparisons for the primary efficacy outcome. Multiple administrations, when not delivered following a 
fixed schedule, will contribute to the 'individuals needing additional interventions' outcome. We will include only study arms in which individuals have been randomised to drugs within the recommended dose range for aggressive or agitated individuals with psychosis. ${ }^{30}$ Should these not be available in the current literature, we will consider licensed dose ranges for psychiatric or, if absent, medical conditions. If a study included arms with both approved and unapproved doses, we will include only those arms that employed eligible doses. Should a comparison include different dosages of the same drug, we will consider them as a single node as long as the dosages are within the eligible range. Therefore, studies comparing the same drug at different dosages without another intervention will not contribute the network. For studies with a fixed dosing strategy, we will consider the planned dosage as the administered one. If a study employs a flexible dosing strategy, we will abide by the following hierarchy: (1) mean dose within 48 hours since the first administration; (2) mean dose until the end of the study; (3) average between the minimum and maximum doses allowed. Fairness of dose comparisons in head-to-head trials will be evaluated in a sensitivity analysis. Drug monotherapies and drug combinations within eligible dose ranges will be treated initially as different nodes in the network.

\section{Outcome measures}

Our primary outcomes are:

1. Efficacy (as continuous outcome)-aggression or agitation scores as close to 2 hours as possible since administration of the intervention (range: $>0, \leq 6$ hours). Endpoint scores will be preferred to change scores, if both are reported. We will employ validated rating scales or subscales commonly used to evaluate aggression or agitation including but not limited to Behavioral Activity Rating Scale, ${ }^{31}$ Corrigan Agitated Behavior Scale, ${ }^{32}$ Overt Agitation Severity Scale, ${ }^{33}$ MOAS $^{34}{ }^{35}$ or PANSS-EC. ${ }^{36}$ If more than one scale has been administered, we will prioritise scales measuring aggression over agitation and scales over subscales. It is likely that across included trials different rating scales have been used to measure the same outcome. ${ }^{37}$

To overcome this, we plan to harmonise continuous outcomes to a common metric (ie, standardised mean difference (SMD)). We acknowledge that during a trial additional drugs may be administered as rescue drug to give relief from adverse events. Should this occur, we will use the closest time point to 2 hours after baseline at which additional drugs have not yet been administered. Should this be unavailable, we will include data only if administration of rescue drugs was not unbalanced between the groups (eg, mean value of daily administered milligrams) and we will evaluate the impact of their inclusion in a sensitivity analysis (refer to the Exploring heterogeneity, inconsistency and sensitivity analyses section). If both aggression and agitation data are collected within the same trial at the preferred time points, we will choose the aggression one since it is more representative of clinical urgency. If only agitation outcomes have been measured or reported, we will use this as a proxy of aggression outcome.

2. Tolerability (as dichotomous outcome)-dropouts due to adverse events as close as to 24 hours since the administration of the intervention (range: $>0, \leq 7$ days). Our secondary outcomes include:

1. Efficacy (as continuous outcome)-aggression or agitation scores as close to 24 hours as possible since the administration of the intervention (range: $>6$, $\leq 48$ hours).

2. Efficacy (as dichotomous outcome)-proportion of individuals needing one or more additional interventions (ie, additional administration of any drugs to achieve tranquillisation, needing restraints or seclusion) up to 6 hours since the administration of the intervention (range: $>0, \leq 6$ hours). When the number of individuals in need of additional treatment is reported specifically for every adjunctive care option, summing these data may lead to double counting issues (ie, individuals who needed two or more different additional interventions). Hence, we will abide by the following order: (1) additional administration of any drugs, (2) needing restraints and (3) needing seclusion.

3. Efficacy (as dichotomous outcome)-proportion of individuals needing an additional intervention (ie, additional administration of any drugs to achieve tranquillisation, needing restraints or seclusion) up to 48 hours since the administration of the intervention (range: $>0, \leq 48$ hours). Outcomes will be prioritised as stated above.

4. Tolerability (as dichotomous outcome)-proportion of individuals with at least one serious side effect as close to 24 hours as possible since the administration of the intervention (range: $>0, \leq 7$ days). We define 'serious' side effects as stated by the original investigators.

For the efficacy outcomes, should two available measurements be equidistant from the predefined time point we will prioritise earlier time points within the above stated range. For the tolerability outcomes, should the same conditions apply we will prefer later estimates over the earlier if both are eligible.

\section{Search strategy}

We will search the Cochrane Schizophrenia Group register for studies relevant to aggression and agitation in schizophrenia spectrum illnesses. In this study-based register, searching the major concept retrieves all the synonyms and relevant studies because all the studies have already been organised based on their interventions and linked to the relevant topics. This allows rapid and accurate searches that reduce waste in the next steps of systematic reviewing. ${ }^{38}$ This register is compiled by systematic searches of major resources (AMED, 1985-present; BIOSIS, 1969-present; CENTRAL, not applicable; CINAHL, 1982-present; ClinicalTrials. Gov, 2000-present; Embase, 1974-present; ISRCTN, 2000-present; MEDLINE, 1946-present; PsycINFO, 
1806-present; PubMed, 1946-present; WHO ICTRP, 2000-present) and their monthly updates, ProQuest Dissertations and Theses A\&I (1637-present) and its quarterly update, Chinese databases (CNKI, 1994present; SinoMed, 1978-present; VIP, 1989-present; Wanfang, unclear-present) and their annual updates, hand-searches, grey literature and conference proceedings. ${ }^{40}$ There is no language, date, document type, or publication status limitations for inclusion of records into the register. An additional search on CENTRAL (not applicable), Embase (1974-present) and PubMed (1946present) databases will be performed with keywords relevant to psychotic depression, episodes of bipolar mania or depression with psychosis, delusional disorder and psychotic symptoms, combined with condition-specific (eg, aggression, agitation) and study type-specific (eg, random, RCT) terms. We plan no restrictions in terms of language, country or publication period for this search. We will also inspect similar systematic or non-systematic reviews as additional sources of potentially eligible trials. Search queries, dates and number of identified records will be published in the supplementary material and depicted in a PRISMA flowchart.

\section{Studies selection}

Two members of the team will independently review any identified record. In case of disagreement, the record will be included to assess its eligibility at the full-text phase. Two members of the team will independently review the full text of retrieved potentially eligible studies. We will develop a checklist of eligibility criteria to ease the systematic assessment of the retrieved full texts. We will resolve any disagreement by discussion with a third member of the team. Identified records referring to the same trial (ie, secondary publication) will be collated. We will classify trials according to the Cochrane Handbook definitions in four mutually exclusive categories (ie, included, excluded, awaiting assessment, ongoing) ${ }^{41}$ We will list the reasons for exclusion and any other relevant comment in the supplementary material. We will contact original investigators of the trials to retrieve any additional information deemed useful. In case of no response, we will send weekly reminders up to 3 weeks (ie, three reminders). We will report in the supplementary material any attempt to contact original investigators and the extent of their contribution (eg, information and/or data provided, no answer).

\section{Data extraction}

We will design and use a structured data extraction form to ensure consistency of extracted information and data. We will extract the following data:

- Methods: date of the study, study design, randomisation (ie, individual or cluster), number of study centres.

- Participants: number, proportion of first-episode patients, gender, age, underlying treatments, inclusion and exclusion criteria, co-morbid disorders.
- Interventions: dose, dose range, dosing strategy, frequency of administration, route of administration, use of rescue medication.

- Outcomes: definition, measures, timing of assessment.

- Others: sponsorship or funding for trial (eg, industry, academic), notable conflict of interests of trial original investigators.

Data estimates retrievable by graphs will be extracted (eg, mean, SD) using Plot Digitizer, an electronic ruler. ${ }^{42}$ If both the SD and the SE are missing but $\mathrm{p}$ values or CIs are available, we will calculate SDs following the Cochrane Handbook guidelines. ${ }^{43}$ Should the original publication provide continuous data relevant to an outcome hereby specified as dichotomous, we will impute response rates from means and SD by employing a validated imputation method. ${ }^{44}$

\section{Missing outcome data}

To overcome the issue of missing outcome data, we aim at replicating the methods previously employed in a recent NMA on depression. ${ }^{45}$ For continuous outcomes, when data for a specific outcome is available for both intention-to-treat and per-protocol populations, preference will be given to the former. We will prefer data based on mixed-effect models of repeated measurements, multiple imputation or any other appropriate imputation methods over last-observation-carried-forward data. $^{46}$ We will exclude participants with missing outcome data from the analysis. For the dichotomous efficacy outcome, we will assume that participants with missing outcome data failed to achieve an adequate response. Dropouts from assessment will be assumed not to have changed from baseline for the given outcome: not in need of additional interventions in terms of efficacy outcomes, and not having developed an adverse effect in terms of tolerability outcomes. We will evaluate the appropriateness of the imputation method in the Risk of bias judgement section.

\section{Unit of analysis issues}

We will extract data from cross-over studies only for the first phase (ie, before cross-over) to avoid including data potentially affected by the carry-over effect. However, given that we will extract data from relatively short trials, we expect cross-over effect to be unlikely. In trials that employ 'cluster randomisation' (eg, by practice or clinician), we will extract data adjusted for the clustering effect. If these are not available, we will extract unadjusted data and divide the sample size (for continuous outcomes) or both sample size and number of events (for dichotomous outcomes) by the design effect, defined as follows ${ }^{47}$ :

$$
\text { Design effect }=1+(m-1) \text { ICC }
$$

where $m$ denotes the mean number of participants per cluster and ICC is the intra-cluster correlation coefficient, an estimate of the relative variability within and between clusters. ${ }^{48}$ If the ICC is not reported, we will assume it to be $0.1 .^{49}$ 


\section{Comparability of dosages}

Some trials may have employed unfair dose comparisons, possibly biasing the resulting estimate. To overcome this, we will add a dichotomous variable to indicate whether the comparison was fair by narrowing the eligible range of doses (ie, excluding lower and upper $20 \%$ of doses of the recommended or licensed ranges). Should a combination include two or more drugs at different levels of the range of doses, we will rate the combination according to the drug at the higher one (ie, hierarchically as upper range, mid-range, lower range).

\section{Risk of bias judgement}

To evaluate the risk of bias, we will use the revised Cochrane risk of bias tool for randomised trials (RoB 2) (V.9, July 2019).$^{50}$ Two independent raters will perform the evaluation following the full guidance document and using the provided Excel tool. In case of disagreement, consensus will be reached either by discussion or by consultation of a third member of the team. Studies contributing to least one primary outcome will be assessed in the following domains: bias arising from the randomisation process, bias arising from identification or recruitment of individual participants within clusters (only for cluster-randomised trials), bias due to deviations from intended interventions (effect of assignment to the interventions at baseline), bias due to missing outcome data, bias in measurement of the outcome, bias in selection of the reported result.

Should a study contribute to both primary outcomes, we will provide two outcome-specific separate risk of bias judgements.

\section{Additional domains}

Additionally, we will evaluate the influence of sponsorship and notable conflict of interest, defined as follow:

- Sponsorship (arm-level): (1) funded by a pharmaceutical company with an active interest or (2) one or more authors were employees of such company.

- Notable conflict of interest (arm-level): (1) funded by a pharmaceutical company with an active interest, (2) one or more authors were employees of a company with an active interest, (3) having received speaker fees or honoraria from a company with an active interest or (4) having disclosed any other notable conflict of interest.

These two domains will not contribute towards the overall risk of bias judgement but will be separately addressed (refer to the Exploring heterogeneity, inconsistency and sensitivity analyses section).

\section{Overall risk of bias judgement}

Studies will be classified according to the guidance manual for RoB $2^{50}$ :

- Overall 'low risk of bias' if all the considered domains are rated to be at low risk of bias.

- Overall 'some concerns' if (1) at least one of the considered domains is rated to raise concern and (2) none of the considered domains is rated to be high risk of bias.

- Overall 'high risk of bias' if (1) at least one of the considered domains is rated to be at high risk of bias (irrespective of which domain is being assessed) or (2) the study is judged to raise concerns in multiple domains.

\section{Statistical synthesis of study data}

Characteristics of included studies and information flow in the network

We will provide descriptive statistics for the characteristics of trial and study population across eligible trials and also visualise relevant information by producing a network diagram. To understand which comparison contributes the most to the network estimate and how it affects the estimate, the percentage contribution of each direct meta-analysis will be described in a contribution matrix table. ${ }^{51}$

\section{Pairwise meta-analyses}

We will synthesise data of each pairwise comparison to obtain SMDs for continuous outcomes and ORs for dichotomous outcomes, and their 95\% CIs. Assuming that different studies are measuring different but related treatment effects, we will use a random-effects model. We will compare the estimated distribution of the heterogeneity variance $\tau^{2}$ to predictive distributions, as described elsewhere. ${ }^{52} 53$

\section{Assessment of the transitivity assumption}

The transitivity assumption is the basic premise of a NMA. Distribution of clinical and methodological variables likely to act as effect modifiers will be investigated. ${ }^{5455} \mathrm{We}$ assume that individuals who fulfil the inclusion criteria are, in principal, equally likely to be randomised to any of the interventions of interest (ie, jointly randomisable). When additional evidence of intransitivity is lacking and potential effect modifiers have similar distribution across the included studies, NMA is likely to give valid results. We acknowledge that aggression and agitation might be conceived as different behavioural aspects. However, we will assume that individuals with psychotic symptoms and high levels of either aggression or agitation will have a similar clinical presentation and will receive similar interventions. Aggression or agitation due to psychotic symptoms induced by substance or medication use or due to a medical condition may moderate efficacy and tolerability of rapid tranquillisation interventions. For instance, antipsychotics are usually preferred over benzodiazepines in individuals with alcohol intoxication because of the risk of respiratory depression, while for other substances interventions other than antipsychotics are often prioritised to avoid increasing cardiotoxicity risk. ${ }^{56}$ Despite this, clinical presentation of these conditions in the acute setting may be difficult to distinguish. To overcome this, we restricted eligible studies to those focusing (ie, $\geq 50 \%$ of total recruited sample) on individuals with psychosis 
not induced by external causes or medical conditions. We do not expect these last to be an effect modifier of efficacy or tolerability of rapid tranquillisation interventions. Therefore, inclusion of diagnoses other than schizophrenia should not threat the plausibility of our transitivity assumption.

Other clinical or methodological variables that may influence our primary outcomes are year of study, age, baseline severity of aggression or agitation, baseline severity of mental state, comorbid substance or alcohol abuse (secondary diagnosis). We will investigate the distribution of these variables across studies grouped by comparison.

\section{Network meta-analyses}

If undertaking NMA is deemed appropriate as described above, we will perform a joint synthesis of all trials per outcome. Network diagrams for all the included studies and for the subsets contributing each outcome will be produced. For dichotomous outcomes of rare events, we will perform a common-effect Mantel-Haenszel NMA. ${ }^{57}$ For all other outcomes, we will employ a random-effects NMA assuming a single heterogeneity parameter for all comparisons in each network. We will present summary estimates (ie, SMD, OR) for all pairwise comparisons in a league table. We will also provide the prediction intervals to assess how much the common heterogeneity affects the relative effect with respect to the additional uncertainty anticipated in a future study. We will calculate the probability for each drug to be ranked at each possible place and will present them in rankograms. ${ }^{58}$ To assess how likely it is for each drug to 'beat' most of its competitors in each outcome, we will calculate the SUCRA and the mean rank for each drug per outcome. ${ }^{58}$

\section{Assessment of inconsistency}

The statistical manifestation of transitivity is termed consistency, which relies on agreement between direct and indirect estimates. ${ }^{25}$ Since consistency can be assessed only in the presence of both direct and indirect evidence (ie, closed loop), when these are absent-by definitionthere cannot be inconsistency. Absence of statistical inconsistency never ensures the validity of transitivity, which itself remains an assumption. ${ }^{59}$ When closed loops are present in the network, inconsistency may result from variation of effect modifiers across treatment comparisons. ${ }^{60}$ To evaluate inconsistency, we will employ both local and global methods. ${ }^{545}$ We will employ the 'separating indirect from direct evidence approach', which separates direct evidence from indirect evidence to evaluate local consistency, ${ }^{59} 61$ and the design-by-treatment interaction test to evaluate consistency in the entire network. ${ }^{62} 63$

\section{Exploring heterogeneity, inconsistency and sensitivity analyses}

We expect small amounts of heterogeneity and inconsistency to be present. We will perform the following subgroup analyses for our two primary outcomes:

1. Sponsorship/notable conflict of interest.
2. Arms with a proportion equal to or greater than $50 \%$ of participants with comorbid substance-use disorders.

3. Route of administration. In case of combined treatment with different routes of administration, we will classify it according to the drug at the higher level of range of doses (ie, hierarchically as upper range, midrange, lower range). Should it be the same, we will classify it as 'intramuscular'.

We will also perform the following sensitivity analyses on the primary outcomes:

1. Blinding (ie, double-blind vs non double-blind).

2. Only studies with balanced dosages, by excluding arms with eligible but unfair dose comparison.

3. Only studies with an overall low risk of bias.

4. Contribution of combining drugs. If feasible, we will perform a component NMA assuming an additive contribution of single drugs to drug combinations (ie, drug A and drug B to estimate the combination $\mathrm{AB}$ ), and no interaction between the two. In this sensitivity analysis, we will group together all the benzodiazepine interventions both for single drugs and drug combinations.

5. Administration of rescue drugs to evaluate the impact of including in the network studies in which rescue drugs were allowed but whose administration was not unbalanced.

6. Studies with specific inclusion criteria for agitation or aggression, by excluding studies with baseline mean levels suggesting the need for an intervention.

\section{Selection bias}

We will use comparison-adjusted and contour-enhanced funnel plots to explore whether results differ between imprecise and more precise trials. ${ }^{5164}$

\section{Model implementation}

We will fit all models in $\mathrm{R}$. We will use the meta package to perform pairwise meta-analyses. ${ }^{65}$ All the random-effects NMA will be fit using the netmeta package assuming a common $\tau^{2}{ }^{66}$

\section{Assessment of the confidence in the evidence from NMA}

To assess the confidence we can place in the relative treatment effects estimated in NMA, we will use the Confidence in Network Meta-Analysis (CINeMA) framework, implemented in the web application https://cinema. ispm.unibe.ch. CINeMA evaluates the credibility of the findings across the following domains: within-study bias, across-studies bias, indirectness, imprecision, heterogeneity and incoherence. ${ }^{67} 68$ Imprecision, heterogeneity and incoherence domains require to specify a clinically important size of effect on the scale of the considered effect measure. We will set the following margin of non-equivalence: (1) active versus active comparison, a SMD of 0.1 for continuous outcome (severity of aggression/agitation) and an OR of 2.0 for dichotomous outcome (dropouts due to adverse events). We will apply these criteria to both active versus active and active versus 
placebo comparisons, since we are mainly interested in relative effect of active treatments.

\section{Patient and public involvement}

No patient was involved in the protocol development nor will be involved in the review.

\section{ETHICS AND DISSEMINATION}

This study does not require ethical approval. We will disseminate our findings by publishing results in a peer-reviewed journal.

\section{Author affiliations \\ ${ }^{1}$ Department of Health Sciences, Università degli Studi di Milano, Milan, Italy ${ }^{2}$ Cochrane Schizophrenia Group, Division of Psychiatry and Applied Psychology, Institute of Mental Health, School of Medicine, University of Nottingham, Nottingham, UK \\ ${ }^{3}$ Institute of Social and Preventive Medicine, University of Bern, Bern, Switzerland ${ }^{4}$ Department of Health Promotion and Human Behavior and Department of Clinical Epidemiology, Graduate School of Medicine and School of Public Health, Kyoto University, Kyoto, Japan}

\section{Twitter Farhad Shokraneh @Farhad_Cochrane and Toshi A Furukawa @Toshi_ FRKW}

Contributors EGO, TAF and GS conceived the study. EGO, TAF, GS, FS and ADA provided substantial contribution to the design of the study during its development. EGO, TAF, GS, FS and ADA revised the study protocol critically for important intellectual content and contributed to its improvement. FS have planned the search in Cochrane Schizophrenia Group (CSzG) register and will conduct it. EGO have planned the search outside the CSzG register and will conduct it. GS supervised the statistical analysis planning. EGO, TAF, GS, FS and ADA gave the final approval to publish the current protocol. All the authors agreed to be accountable for all aspects of the work in ensuring that questions related to the accuracy or integrity of any part of the work are appropriately investigated and resolved.

Funding The authors have not declared a specific grant for this research from any funding agency in the public, commercial or not-for-profit sectors.

Competing interests GS was invited to participate in two scientific meetings on the use of real-world evidence organised by Merck and Biogen. TAF reports personal fees from Meiji, Mitsubishi-Tanabe, MSD and Pfizer and a grant from Mitsubishi-Tanabe, outside the submitted work; TAF has a patent 2018-1 77688 pending.

Patient consent for publication Not required.

Provenance and peer review Not commissioned; externally peer reviewed.

Open access This is an open access article distributed in accordance with the Creative Commons Attribution Non Commercial (CC BY-NC 4.0) license, which permits others to distribute, remix, adapt, build upon this work non-commercially, and license their derivative works on different terms, provided the original work is properly cited, appropriate credit is given, any changes made indicated, and the use is non-commercial. See: http://creativecommons.org/licenses/by-nc/4.0/.

\section{ORCID iDs}

Edoardo G 0stinelli http://orcid.org/0000-0002-8717-0832

Armando D'Agostino http://orcid.org/0000-0002-2126-799X

Farhad Shokraneh http://orcid.org/0000-0001-9687-8560

\section{REFERENCES}

1 Larkin GL, Claassen CA, Emond JA, et al. Trends in U.S. emergency department visits for mental health conditions, 1992 to 2001. PS 2005;56:671-7.

2 New A, Tucci VT, Rios J. A modern-day fight Club? The stabilization and management of acutely agitated patients in the emergency department. Psychiatr Clin North Am 2017;40:397-410.

3 Mohr P, Pecenák J, Svestka J, et al. Treatment of acute agitation in psychotic disorders. Neuro Endocrinol Lett 2005;26:327-35.
4 Nordstrom K, Allen MH. Managing the acutely agitated and psychotic patient. CNS Spectr 2007;12:5-11.

5 Witt K, van Dorn R, Fazel S. Risk factors for violence in psychosis: systematic review and meta-regression analysis of 110 studies. PLoS One 2013;8:e55942

6 Onwumere J, Grice S, Garety P, et al. Caregiver reports of patientinitiated violence in psychosis. Can J Psychiatry 2014;59:376-84.

7 Smith LM, Onwumere J, Craig T, et al. Caregiver correlates of patient-initiated violence in early psychosis. Psychiatry Res 2018;270:412-7.

8 Ashton RA, Morris L, Smith I. A qualitative meta-synthesis of emergency department staff experiences of violence and aggression. Int Emerg Nurs 2018;39:13-19.

9 Hodgins S, Alderton J, Cree A, et al. Aggressive behaviour, victimisation and crime among severely mentally ill patients requiring hospitalisation. Br J Psychiatry 2007;191:343-50.

10 Lukens TW, Wolf SJ, Edlow JA, et al. Clinical policy: critical issues in the diagnosis and management of the adult psychiatric patient in the emergency department. Ann Emerg Med 2006;47:79-99.

11 National Institute for Clinical Excellence (NICE). Violence and aggression: short-term management in mental health, health and community settings, 2015. Available: nice.org.uk/guidance/ng10 [Accessed Jun 2019].

12 Ahmed U, Jones $\mathrm{H}$, Adams CE, et al. Chlorpromazine for psychosis induced aggression or agitation. Cochrane Database Syst Rev 2010;14.

13 Belgamwar RB, Fenton M. Olanzapine Im or velotab for acutely disturbed/agitated people with suspected serious mental illness. Cochrane Database Syst Rev 2005;2.

14 Berk M, Rathbone J, Mandriota-Carpenter SL, et al. Clotiapine for acute psychotic illnesses. Cochrane Database Syst Rev 2004;11.

15 Huf G, Alexander J, Gandhi P, et al. Haloperidol plus promethazine for psychosis-induced aggression. Cochrane Database Syst Rev 2016;11.

16 Jayakody K, Gibson RC, Kumar A, et al. Zuclopenthixol acetate for acute schizophrenia and similar serious mental illness. Cochrane Database Syst Rev 2012;4.

17 Khokhar MA, Rathbone J. Droperidol for psychosis-induced aggression or agitation. Cochrane Database Syst Rev 2016;12.

18 Khushu A, Powney MJ. Haloperidol for long-term aggression in psychosis. Cochrane Database Syst Rev 2016;11.

19 Ostinelli EG, Brooke-Powney MJ, Li X, et al. Haloperidol for psychosis-induced aggression or agitation (rapid tranquillisation). Cochrane Database Syst Rev 2017;7.

20 Ostinelli EG, Jajawi S, Spyridi S, et al. Aripiprazole (intramuscular) for psychosis-induced aggression or agitation (rapid tranquillisation) Cochrane Database Syst Rev 2018;1.

21 Ostinelli EG, Hussein M, Ahmed U, et al. Risperidone for psychosisinduced aggression or agitation (rapid tranquillisation). Cochrane Database Syst Rev 2018;4.

22 Vangala R, Ahmed U, Ahmed R, et al. Loxapine inhaler for psychosisinduced aggression or agitation. Cochrane Database Syst Rev $2012 ; 12$.

23 Wilkie F, Fenton M, Cochrane Schizophrenia Group. Quetiapine for psychosis-induced aggression or agitation. Cochrane Database Syst Rev 2012;313.

24 Zaman H, Sampson SJ, Beck AL, et al. Benzodiazepines for psychosis-induced aggression or agitation. Cochrane Database Syst Rev 2017;12.

25 Cipriani A, Higgins JPT, Geddes JR, et al. Conceptual and technical challenges in network meta-analysis. Ann Intern Med 2013;159:130-7.

26 Moher D, Shamseer L, Clarke M, et al. Preferred Reporting Items for Systematic Review and Meta-Analysis Protocols (PRISMA-P) 2015 statement. Syst Rev 2015;4:1.

27 Chaimani A, Caldwell DM, Li T, et al. Additional considerations are required when preparing a protocol for a systematic review with multiple interventions. J Clin Epidemiol 2017;83:65-74.

28 Hutton B, Salanti G, Caldwell DM, et al. The PRISMA extension statement for reporting of systematic reviews incorporating network meta-analyses of health care interventions: checklist and explanations. Ann Intern Med 2015;162:777-84.

29 Shamseer L, Moher D, Clarke M, et al. Preferred Reporting Items for Systematic Review and Meta-Analysis Protocols (PRISMA-P) 2015 elaboration and explanation. BMJ 2015;349:g7647.

30 Gardner DM, Murphy AL, O'Donnell H, et al. International consensus study of antipsychotic dosing. AJP 2010;167:686-93.

31 Swift RH, Harrigan EP, Cappelleri JC, et al. Validation of the behavioural activity rating scale (BARS) ${ }^{\mathrm{TM}}$ : a novel measure of activity in agitated patients. J Psychiatr Res 2002;36:87-95. 
32 Corrigan JD. Development of a scale for assessment of agitation following traumatic brain injury. J Clin Exp Neuropsychol 1989;11:261-77.

33 Yudofsky SC, Kopecky HJ, Kunik M, et al. The overt agitation severity scale for the objective rating of agitation. J Neuropsychiatry Clin Neurosci 1997;9:541-8.

34 Yudofsky SC, Silver JM, Jackson W, et al. The overt aggression scale for the objective rating of verbal and physical aggression. Am J Psychiatry 1986;143:35-9.

35 Kay SR, Wolkenfeld F, Murrill LM. Profiles of aggression among psychiatric patients. I. nature and prevalence. J Nerv Ment Dis 1988;176:539-46.

36 Montoya A, Valladares A, Lizán L, et al. Validation of the excited component of the positive and negative syndrome scale (PANSSEC) in a naturalistic sample of 278 patients with acute psychosis and agitation in a psychiatric emergency room. Health Qual Life Outcomes 2011;9:18.

37 Miyar J, Adams CE. Content and quality of 10000 controlled trials in schizophrenia over 60 years. Schizophr Bull 2013;39:226-9.

38 Shokraneh F, Adams CE. Study-based registers of randomized controlled trials: starting a systematic review with data extraction or meta-analysis. Biolmpacts 2017;7:209-17.

39 Shokraneh F, Adams CE. Study-based registers reduce waste in systematic reviewing: discussion and case report. Syst Rev 2019;8:129.

40 Shokraneh F. Register of trials, Cochrane Schizophrenia Group. Available: https://schizophrenia.cochrane.org/register-trials [Accessed Jun 2019].

41 Clarke M, Oxman AD, Paulsen E. Appendix A: Guide to the contents of a Cochrane Methodology protocol and review. In: Higgins JPT, Green S, eds. Cochrane Handbook for systematic reviews of interventions (version 5.1.0). The Cochrane Collaboration, 2011.

42 Jelicic Kadic A, Vucic K, Dosenovic S, et al. Extracting data from figures with software was faster, with higher interrater reliability than manual extraction. J Clin Epidemiol 2016;74:119-23.

43 Higgins JPT, Deeks JJ. Chapter 7: Selecting studies and collecting data. In: Higgins JPT, Green S, eds. Cochrane Handbook for systematic reviews of interventions (version 5.1.0). The Cochrane Collaboration, 2011.

44 Furukawa TA, Cipriani A, Barbui C, et al. Imputing response rates from means and standard deviations in meta-analyses. Int Clin Psychopharmacol 2005;20:49-52.

45 Furukawa TA, Salanti G, Atkinson LZ, et al. Comparative efficacy and acceptability of first-generation and second-generation antidepressants in the acute treatment of major depression: protocol for a network meta-analysis. BMJ Open 2016;6:e010919.

46 Little RJ, D'Agostino R, Cohen ML, et al. The prevention and treatment of missing data in clinical trials. $N$ Engl $\mathrm{J}$ Med 2012;367:1355-60.

47 Higgins JPT, Deeks JJ, Altman DG. Chapter 16: Special topics in statistics. In: Higgins JPT, Green S, eds. Cochrane Handbook for systematic reviews of interventions (version 5.1.0. The Cochrane Collaboration, 2011.

48 Donner A, Klar N. Issues in the meta-analysis of cluster randomized trials. Stat Med 2002;21:2971-80.

49 Ukoumunne OC, Gulliford MC, Chinn S, et al. Methods for evaluating area-wide and organisation-based interventions in health and health care: a systematic review. Health Technol Assess 1999;3:1-75.
50 Sterne JAC, Savović J, Page MJ, et al. Rob 2: a revised tool for assessing risk of bias in randomised trials. BMJ. In Press;2.

51 Furukawa TA, Miura T, Chaimani A, et al. Using the contribution matrix to evaluate complex study limitations in a network metaanalysis: a case study of bipolar maintenance pharmacotherapy review. BMC Res Notes 2016;9:218.

52 Turner RM, Jackson D, Wei Y, et al. Predictive distributions for between-study heterogeneity and simple methods for their application in Bayesian meta-analysis. Stat Med 2015;34:984-98.

53 Rhodes KM, Turner RM, Higgins JPT. Predictive distributions were developed for the extent of heterogeneity in meta-analyses of continuous outcome data. J Clin Epidemiol 2015;68:52-60.

54 Salanti G, Higgins JPT, Ades AE, et al. Evaluation of networks of randomized trials. Stat Methods Med Res 2008;17:279-301.

55 Salanti G. Indirect and mixed-treatment comparison, network, or multiple-treatments meta-analysis: many names, many benefits, many concerns for the next generation evidence synthesis tool. Res Synth Methods 2012;3:80-97.

56 Ricaurte GA, McCann UD. Recognition and management of complications of new recreational drug use. Lancet 2005;365:2137-45

57 Efthimiou O, Rücker G, Schwarzer G, et al. Network meta-analysis of rare events using the Mantel-Haenszel method. Stat Med 2019;38:2992-3012.

58 Salanti G, Ades AE, loannidis JPA. Graphical methods and numerical summaries for presenting results from multipletreatment meta-analysis: an overview and tutorial. $J$ Clin Epidemiol 2011;64:163-71.

59 Efthimiou O, Debray TPA, van Valkenhoef G, et al. GetReal in network meta-analysis: a review of the methodology. Res Synth Methods 2016;7:236-63.

60 Jansen JP, Naci $H$. Is network meta-analysis as valid as standard pairwise meta-analysis? it all depends on the distribution of effect modifiers. BMC Med 2013;11:159.

61 Dias S, Welton NJ, Caldwell DM, et al. Checking consistency in mixed treatment comparison meta-analysis. Stat Med 2010;29:932-44.

62 Higgins JP, Jackson D, Barrett JK, et al. Consistency and inconsistency in network meta-analysis: concepts and models for multi-arm studies. Res Synth Methods 2012;3:98-110.

63 Jackson D, Barrett JK, Rice S, et al. A design-by-treatment interaction model for network meta-analysis with random inconsistency effects. Stat Med 2014;33:3639-54.

64 Peters JL, Sutton AJ, Jones DR, et al. Contour-enhanced metaanalysis funnel plots help distinguish publication bias from other causes of asymmetry. J Clin Epidemiol 2008;61:991-6.

65 Schwarzer G. Meta: an R package for meta-analysis. $R$ News 2007;7:40-5.

66 Rücker G, Krahn U, König J, et al. netmeta: network meta-analysis using frequentist methods, 2019. Available: https://CRAN.R-project. org/package =netmeta [Accessed Jun 2019].

67 Confidence in Network Meta-Analysis (CINeMA). Institute of social and preventive medicine, University of Bern, 2017. Available: cinema. ispm.ch [Accessed Jun 2019].

68 Salanti G, Del Giovane C, Chaimani A, et al. Evaluating the quality of evidence from a network meta-analysis. PLoS One 2014;9:e99682. 